\title{
L'émergence des entreprises sociales rurales communautaires au Japon
}

The new wave of social enterprises in rural communities in Japan

\section{La emergencia de las empresas sociales rurales comunitarias en Japón}

\section{Matsuyo Makion et Ken-Ichi Kitajima}

Numéro 341, juillet 2016

\section{Asie}

Asia

URI : https://id.erudit.org/iderudit/1037436ar

DOI : https://doi.org/10.7202/1037436ar

Aller au sommaire du numéro

Éditeur(s)

Association Recma

ISSN

1626-1682 (imprimé)

2261-2599 (numérique)

Découvrir la revue

Citer cet article

Makion, M. \& Kitajima, K.-I. (2016). L'émergence des entreprises sociales rurales communautaires au Japon. Revue internationale de l'économie sociale, (341), 44-63. https://doi.org/10.7202/1037436ar
Résumé de l'article

Le Japon a un mouvement coopératif très dynamique, qui s'est traditionnellement développé à partir de deux modèles : celui de la coopérative agricole et celui de la coopérative de consommateurs. A ces deux modèles sont venues ensuite s'ajouter de nouvelles formes de coopératives inspirées par des valeurs différentes, telles que la coopérative de travailleurs ou la " coopérative communautaire ", à laquelle cet article est consacré. Incarnant une forme d'entreprise sociale apparue en milieu rural pour répondre à des besoins ressentis par une communauté, ces coopératives partagent des caractéristiques communes avec les organisations du secteur à but non lucratif. Cet article analyse les origines historiques de ces organisations à partir de démarches apparues dans les années 1970, à l'initiative de producteurs et de consommateurs désirant promouvoir une agriculture biologique, jusqu'aux développements plus récents engagés dans des problématiques liées au développement communautaire, souvent en lien avec des collectivités locales. 


\title{
L'ÉMERGENCE DES ENTREPRISES SOCIALES RURALES COMMUNAUTAIRES AU JAPON
}

\author{
par Matsuyo Makion* et Ken-Ichi Kitajima**
}

Le Japon a un mouvement coopératif très dynamique, qui s'est traditionnellement développé à partir de deux modèles: celui de la coopérative agricole et celui de la coopérative de consommateurs. A ces deux modèles sont venues ensuite s'ajouter de nouvelles formes de coopératives inspirées par des valeurs différentes, telles que la coopérative de travailleurs ou la "coopérative communautaire", à laquelle cet article est consacré. Incarnant une forme d'entreprise sociale apparue en milieu rural pour répondre à des besoins ressentis par une communauté, ces coopératives partagent des caractéristiques communes avec les organisations du secteur $\grave{a}$ but non lucratif. Cet article analyse les origines historiques de ces organisations à partir de démarches apparues dans les années 1970, à l'initiative de producteurs et de consommateurs désirant promouvoir une agriculture biologique, jusqu'aux développements plus récents engagés dans des problématiques liées au développement communautaire, souvent en lien avec des collectivités locales.

\section{The new wave of social enterprises in rural communities in Japan}

Japan has a very dynamic cooperative movement that was traditionally based on agricultural and consumer cooperatives. These were followed by new forms of cooperatives motivated by different values, such as worker cooperatives and "community cooperatives", which are the subject of this article. As a form of social enterprise in rural areas to meet the needs of local communities, these cooperatives have features in common with organisations in the non-profit sector. This article examines the historical origins of these organisations beginning with projects started in the 1970s by producers and consumers interested in promoting organic farming through to more recent initiatives involved in local development issues often in conjunction with local communities.

\section{La emergencia de las empresas sociales rurales comunitarias en Japón}

En Japón, un movimiento cooperativo muy dinámico se ha expandido de manera tradicional a partir de dos modelos: la cooperativa agrícola y la cooperativa de consumidores. A estos dos modelos se han añadido nuevas formas de cooperativas inspiradas en valores diferentes como la cooperativa de trabajadores o la "cooperativa comunitaria" a la que se refiere el presente artículo. Encarnando una forma de empresa social surgida en entorno rural para responder a las necesidades de una comunidad, estas cooperativas comparten características comunes con las organizaciones del sector no lucrativo. Este artículo analiza los orígenes históricos de estas organizaciones a partir de iniciativas iniciadas en los años 70s por productores y consumidores para promover una agricultura biológica, hacia la aplicación de orientaciones comunitarias más recientes, a menudo en relación con comunidades locales

\footnotetext{
*Université de Kansai Gaidai, Osaka, Japon. Mél.: mmakino@kansaigaidai.jp.

** Université de Rikkyo, Tokyo, Japon. Mél. : kitajima@rikkyo.ac.jp.

Traduit par Sihem Mammar El Hadj et révisé par Patricia Toucas-Truyen.
} 
(1) Cadre selon lequel l'entreprise sociale se définit sur la base d'un idéal-type caractérisé par neuf critères, réarticulés en trois composantes: critères économiques, critères sociaux, critères de gouvernance. Pour en savoir plus, lire Defourny et Nyssens (2010).
$\mathbf{L}$ 'histoire des coopératives et du mouvement coopératif au Japon est très ancienne. Leur popularité, qui se vérifie toujours aujourd'hui, date des années 1960-1970. La notion d' "économie sociale ", introduite par l'expérience européenne à partir des années 1990, s'est ensuite diffusée auprès des chercheurs et des praticiens du secteur coopératif. Les coopératives orientées vers le social et l'environnement, telles que les coopératives d'activité et d'emploi et les coopératives de consommateurs écologiquement responsables, ont progressivement développé un sentiment collectif d'appartenance à l'économie sociale et solidaire. Certaines s'identifient en tant qu'entreprise sociale. Le plus souvent, le qualificatif de « coopérative non lucrative» est employé afin d'accentuer l'orientation d'« intérêt général » de l'organisation. Il s'agit là d'aspects nouveaux qui les distinguent des organisations classiques de l'économie sociale telles que les coopératives d'agriculteurs ou de consommateurs.

L'objectif de cette étude est d'identifier l'émergence des entreprises sociales communautaires dans les régions rurales du Japon et de les définir à la lumière du cadre analytique du réseau de recherche européen sur l'économie sociale et solidaire EMES ${ }^{(1)}$. Cet article s'appuie sur l'abondante littérature académique, les rapports et les études de cas publiés par les ministères et les autorités préfectorales, sur les publications des instituts de recherche du secteur coopératif ainsi que sur d'autres sources informelles. Nous avons par ailleurs mené des enquêtes de terrain dans les préfectures de Hyogo, Kyoto et Saitama.

Nous avons classé ces entreprises en trois grandes catégories en fonction de leurs origines historiques et de leur raison d'être. La première catégorie regroupe les organisations issues des groupes d'agriculture biologique et des coopératives de producteurs des années 1970 qui, plus tard, ont renforcé leur orientation communautaire. La deuxième regroupe les organisations issues de l'« agriculture communautaire " (Shuraku-einou) de la fin des années 1980-1990 - il s'agit d'une action collective des agriculteurs locaux dans le but de contenir le détournement et l'abandon des terres agricoles. La dernière catégorie regroupe des organisations apparues dans les années 2000 en réponse aux fusions municipales engagées dans tout le pays. Ces structures sont créées à l'initiative de citoyens engagés dans les communautés locales, la plupart en partenariat avec les autorités locales (les municipalités) et structurées autour des « associations communautaires autonomes" (Chiiki jichi soshiki).

Notre première hypothèse est que ce type d'entreprises rurales, profondément enracinées dans la communauté et la tradition coopérative japonaises, forment un modèle d'entreprise sociale que nous pouvons appeler "coopérative communautaire». 
(2) Durant les années 1960, les ménages agricoles à temps partiel ou en activité secondaire sont devenus le groupe le plus important des foyers ruraux, pour lesquels il s'agissait d'une stratégie de subsistance. (3) Les coopératives agricoles d'aprèsguerre, ou Nogyo Kyodo Kumiai (Nokyo), renommées aujourd'hui coopératives agricoles japonaises (AJ), ont été fondées par la loi sur les coopératives agricoles de 1947. Les Nokyo, considérées comme les meilleures formes d'action collective dans un secteur agricole dominé par des fermes de petite taille, fournissent les services fondamentauxà la communauté rurale. Cependant, le modèle propriétaire-cultivateur est devenu inadéquat, du fait de changements structurels majeurs dans l'agriculture et de la diversification des profils professionnels. Au fil du temps, les services financiers sont devenus le business dominant des $\mathrm{AJ}$, et ces activités commerciales principales liées à l'agriculture ont décliné à partir de 1986.

(4) Fukugo-Osen, «the compound or cumulative contamination» (1975), titre du best-seller de la romancière Sawako Ariyoshi. Il constitue la première étude sur l'impact des engrais chimiques, des colorants cancérogènes, du gaz d'échappement des voitures et d'autres agents polluants. Ce livre a eu un impact sur les mouvements d'alimentation alternative au Japon.
C'est à l'initiative de citoyens issus de la communauté partageant l'objectif commun de redynamisation locale que ces coopératives communautaires sont créées et perpétuées. Elles répondent aux besoins de la communauté non satisfaits par le gouvernement, les organisations lucratives et les coopératives classiques. En mobilisant les ressources de la communauté de façon innovante, elles fournissent une variété de «produits communautaires » quasi publics et témoignent de l'évolution du modèle d'autogouvernance ancré au sein de la communauté.

\section{Renouveau du mouvement coopératif dans l'agriculture}

Les origines des entreprises sociales rurales remontent aux années 1970, lorsque l'agriculture de l'après-guerre a commencé à connaître de réels problèmes structurels: stagnation de la productivité, augmentation des inégalités de salaires entre les régions urbaines et rurales, exode urbain des jeunes, pénurie de main-d'œuvre agricole et changements de la structure des revenus des ménages agricoles ${ }^{(2)}$. Le gouvernement et les coopératives agricoles n'ont pas su régler correctement ce problème ${ }^{(3)}$. Les années 1970 ont été aussi la période pendant laquelle le peuple japonais a pris conscience des effets négatifs d'une croissance économique importante sur l'environnement naturel et social. Parallèlement au mouvement social massif contre la pollution industrielle, le mouvement coopératif a connu un nouveau souffle. De nouvelles formes de coopération sont nées, telles que les Teikei dans l'agriculture biologique, les coopératives dédiées à l'alimentation naturelle et les coopératives d'activité. L'approche du " développement endogène ", qui a émergé en Europe, en Amérique du nord et au Japon dans les années 1970 et s'est ensuite enrichie du concept de développement durable, a significativement influencé la recherche et la pratique relatives au développement rural du Japon.

\section{Fermiers entrepreneurs dans l'agriculture biologique et naturelle}

Au début des années 1970, la " pollution mélangée ${ }^{(4)}$ » et les effets négatifs associés à l'« agriculture industrielle » ont considérablement attiré l'attention des agriculteurs et des consommateurs concernés. Des groupes d'entrepreneurs agricoles ont repris les anciennes pratiques d'agriculture naturelle et organique. Il s'agit d'anciens militants des mouvements étudiants ou de repreneurs d'exploitations agricoles qui sont revenus dans leur village natal afin de se consacrer à un nouveau type de progrès technique dans l'agriculture. Leur principale innovation organisationnelle, appelée Teikei, est une sorte d'entente entre agriculteurs locaux et consommateurs responsables, qui existe encore aujourd'hui. 
(5) Selon O’Hara (2011), Teikei est largement répandu aujourd'hui aux Etats-Unis et est décrit comme une "agriculturesoutenue par la communauté» (ASC).
(6) Le club coopératif de Seikatsu, fondé en 1968 à Tokyo, est aussi connu comme organe fondateur des collectifs de travailleurs au Japon (une fédération de coopératives de travailleurs appartenant à des femmes).

(7) AJAB a été fondée en 1971à l'appel de Teruo Ichiraku, leader important du mouvement coopératif et directeur général de l'Institut de recherche en management des coopératives (dénommé aujourd'hui Institut de recherche générale sur les coopératives japonaises [CJ]). Shin-ichi Wakatsuki, médecin et fondateur de l'hôpital général de Saku, dans la préfecture de Nagano, qui est devenu le premier hôpital coopératif dans les régions rurales du Japon, futl'un de ses inspirateurs. (8) Les coopératives de travailleurs sont apparues au Japon au début des années 1970 afin de fournir un emploi aux chômeurs. Elles se sont développées après les chocs pétroliers. Les «collectifs de travailleurs" pour les femmes sont apparus en 1982.
Les consommateurs adhérents s'engagent à payer en avance, au début de la saison, quand la ferme a besoin de financement. En échange, ils reçoivent des envois hebdomadaires de produits frais et sains. Grâce à cette organisation, les agriculteurs passent moins de temps à la commercialisation pendant la saison de culture $^{(5)}$. D'un point de vue économique, cette commercialisation directe permet aux producteurs de contourner les marchés de gros et de détail et d'internaliser la valeur ajoutée créée grâce à ces liens étroits. En outre, cela permet aux consommateurs de remédier à l'asymétrie d'information sur les aliments fournis et d'accéder à des produits de première qualité, tout en soutenant les agriculteurs locaux. D'un point de vue social, ce système facilite la création d'un lien direct, qui rapproche la ville et la campagne par ces échanges entre urbains et ruraux.

Pour partie, la mise en place de ce système était devenue nécessaire pour les agriculteurs biologiques. Ces derniers étaient forcés de trouver un moyen de vendre leurs produits sans dépendre de la coopérative d'agriculture Nokyo (AJ), qui était devenue un promoteur actif de l'agriculture chimique intensive et de la monoculture et qui détenait un quasi-monopole sur la distribution agricole. Le mouvement Teikei a grandement influencé les consommateurs responsables, de plus en plus insatisfaits par leurs coopératives. Certains d'entre eux ont lancé leurs coopératives d'alimentation écologique. L'une des premières, Seikatsu Club (une coopérative de consommateurs dans la métropole de Tokyo), en est un exemple important ${ }^{(6)}$. La plupart de ces entrepreneurs sont restés des exploitants individuels. Cependant, ils ont formé un groupe soudé et étaient membres d'organisations non lucratives telles que l'Association japonaise d'agriculture biologique (AJAB) ${ }^{(7)}$.

\section{Version rurale des coopératives d'activités}

Au cours des années 1970, un groupe de jeunes agriculteurs, pour la plupart des repreneurs d'exploitation, concernés par l'avenir de l'agriculture, adoptent la forme juridique de l'entreprise coopérative de producteurs agricoles (Noji kumiai-hojin). Il s'agit d'un nouveau type de coopérative agricole, fondé en 1962 suite à la loi sur les coopératives agricoles, qui permet de surmonter les limites de l'agriculture familiale. Selon Iwami (2007), ces entreprises coopératives de producteurs agricoles sont une version rurale des coopératives d'activité( ${ }^{(8)}$.

Ces coopératives, souvent engagées dans une agriculture écologique, ont établi des relations fortes avec d'autres nouvelles formes de coopératives dans les régions urbaines, comme les coopératives de consommateurs écologiquement responsables et les coopératives d'activité. En développant leurs échanges avec des consommateurs urbains et en diversifiant leurs activités commerciales à la transformation et à la distribution, 
elles ont rompu leur dépendance vis-à-vis des Nokyo, la forme classique de la coopérative agricole.

Même si le concept d'" entreprise sociale " n'existait pas encore, les entrepreneurs agricoles et les coopératives de producteurs de cette époque présentent des caractéristiques similaires avec les entreprises sociales d'aujourd'hui. En tant qu'" entrepreneurs sociaux » ruraux ou réformistes du secteur coopératif, ils ont instauré une nouvelle façon de créer du lien avec les consommateurs, par la confiance et le soutien mutuel: la coproduction et la distribution d'aliments sains et sûrs par les producteurs et les consommateurs, et le commerce équitable rural-urbain. Ils ont fourni à la société des biens et des services d'intérêt social qui n'étaient pris en charge ni par les entreprises lucratives ni par les coopératives agricoles ou de consommateurs classiques. Ces nouvelles entreprises coopératives de producteurs agricoles sont devenues les précurseurs de l'agriculture communautaire.

\section{Emergence des entreprises sociales rurales communautaires}

Au milieu des années 1980 et au début des années 1990, c'est-à-dire pendant la "bulle économique », les nouveaux mouvements coopératifs se sont affaiblis. L'explosion de cette bulle a précipité l'économie japonaise dans une récession de très longue durée. La globalisation, la baisse des prix des produits agricoles et le déclin de l'industrie manufacturière rurale ont conduit à un déclin important des revenus agricoles et non agricoles. Ces conditions ne permettaient plus aux ménages agricoles individuels de maintenir leur mode de vie traditionnel. De plus, une fois l'émigration stabilisée, le vieillissement et la diminution naturelle de la population sont devenus plus aigus dans les communautés rurales, donnant lieu à ce que l'on nomme la "rétraction communautaire ". La globalisation et la stagnation de l'économie ont cependant permis l'émergence d'un mouvement social, particulièrement dans les régions rurales, ayant pour but la redécouverte de la valeur des traditions et des cultures locales et la réévaluation de la pensée endogène du développement durable des 1970. Dans ce contexte, le nouveau millénaire fut témoin du renouveau communautaire et coopératif.

En 1999, le gouvernement a promulgué une loi fondamentale sur l'alimentation, l'agriculture et les régions rurales qui annonçait de nouveaux objectifs nationaux dans ces domaines: sécurité alimentaire, rôles multifonctionnels de l'agriculture, développement de l'agriculture durable et redynamisation des régions rurales. Le gouvernement a modifié les mesures de soutien aux agriculteurs qui étaient fondées sur des subventions à la production, en instaurant un paiement direct ciblé autour 
(9) 1) Adhésion volontaire et ouverte à tous ; 2) Pouvoir démocratique exercé par les membres; 3) Participation économique des membres ; 4) Autonomie et indépendance ; 5) Education, formation et information; 6) Coopération entreles coopératives ; 7) Engagement envers la communauté. de trois critères: la multifonctionnalité, les plaines défavorisées et les régions montagneuses, l'agriculture durable. Dans les faits, cependant, le gouvernement a priorisé la restructuration du secteur agricole par la consolidation des exploitations agricoles en augmentant la production, au lieu de créer des communautés rurales saines, résilientes et soutenables. De nombreuses entreprises communautaires ou fondées sur la communauté sont nées en tant que réponse ascendante, afin de combler ce vide.

\section{De l'agriculture biologique à l'agriculture soutenue par la communauté}

Lorsque la première génération d'exploitants agricoles biologiques a réussi, les pratiques, les modes de vie et les pensées de ces derniers ont eu une influence importante sur leurs communautés. Les exploitations avoisinantes ont commencé à apprendre de leurs méthodes et se sont parfois engagées dans la transition de l'agriculture classique vers l'agriculture biologique. L'expertise des pionniers a aidé les agriculteurs locaux à développer leurs compétences techniques et leur productivité. Les agriculteurs biologiques, autrefois isolés, sont ainsi devenus des ressources humaines indispensables à la communauté locale. Ces expériences collaboratives ont conduit à l'émergence d'initiatives locales de soutien à l'agriculture biologique, qui, en retour, a amélioré les opportunités de relance de l'économie locale.

Le mouvement ascendant des agriculteurs écologiques a eu une influence importante sur les législateurs dans les années 2000. Ainsi, la loi sur l'agriculture biologique fut promulguée en 2006, ouvrant la voie à une politique intégrée de soutien à l'agriculture biologique. D'autres initiatives locales furent lancées grâce aux mesures gouvernementales telles que la constitution des «villes biologiques ». En 2011, on comptait dans le pays quarante-sept villes de ce type. Un conseil de promotion de l'agriculture biologique fut constitué dans chacune d'entre elles, sous la direction d'un groupe d'agriculteurs biologiques. De nombreuses villes biologiques ont créé une organisationcadre afin de promouvoir une agriculture durable, en collaboration avec la communauté agricole et les organisations non lucratives locales.

Les coopératives de producteurs ruraux les plus avancées, nées en 1970, ont connu le même changement, développant progressivement une agriculture soutenue par la communauté. Leurs activités ont souvent dépassé la dimension économique pour aborder des enjeux environnementaux et sociaux. Cette initiative a été facilitée par les nouveaux principes de l'Alliance coopérative internationale (ACI) ${ }^{(9)}$ définis en 1995 . Au début des années 2000, l'Union des coopératives d'activités du Japon (UCAJ) a fait de la création d'un lien entre alimentation, agriculture et environnement l'une de ses missions principales. 
Yonezawa-Kyo, ferme d'élevage dans la préfecture d'Akita, devenue une grande organisation à la fin des années 1990 en intégrant des coopératives plus petites, a pris la forme d'une entreprise par actions en 2006. Cette ferme a réussi à mettre en place un système de recyclage intégré de fourrage et de fumier simultanément dans l'ensemble du groupe. L'une des organisations du groupe, le club des agriculteurs Akatombo, est présente aujourd'hui dans quatre petites villes et trois villes moyennes. Elle est en recherche active de solutions systématiques aux problèmes de la communauté par le soutien et la formation des entrants potentiels et des échanges ruraux-urbains tels que les visites de fermes, l'expérience agricole et des activités éducatives et sportives. En 2004, la préfecture d'Ehime a mis en place la coopérative communautaire de Muchacha-en (une entreprise par actions), intégrant trois coopératives, avec l'idée de créer une " utopie de fermiers ». Cette coopérative communautaire se chargeait de former les nouveaux entrants à l'agriculture biologique au sein de la communauté, mais aussi à l'étranger (deux au Vietnam). Muchacha-en est également active dans l'intégration dans l'emploi des jeunes et des personnes en situation de handicap, ainsi que dans le maintien de son appartenance à l'UCAJ et à l'Association japonaise d'agriculture biologique. Sa section féminine a commencé son service communautaire (livraison de nourriture) en 2009.

\section{Développement de l'agriculture communautaire APPARITION ET CARACTÉRISTIQUES DE L'AGRICULTURE COMMUNAUTAIRE}

Apparue aux débuts des années 1970, l'agriculture communautaire (Shuraku einou) a gagné en visibilité dans les années 1990, bien qu'elle ne se soit diffusée qu'après les années 2000. Elle a émergé sous le leadership d'agriculteurs avant-gardistes au sein des coopératives agricoles japonaises (AJ). Au niveau préfectoral, les autorités locales, conscientes des problèmes des petites exploitations agricoles, ont évalué positivement le potentiel de l'agriculture communautaire et, de ce fait, ont décidé de soutenir les initiatives des agriculteurs. L'agriculture communautaire s'est développée dans les régions vallonnées et montagneuses, principalement dans les régions de l'ouest du Japon qui sont désavantagées dans l'agriculture intensive du riz et la commercialisation sur les marchés urbains.

Selon la préfecture de Shimane (2002), la définition de l'agriculture communautaire se définit par «des pratiques agricoles dans lesquelles la majorité des ménages agricoles au sein de la communauté sont d'accord sur les objectifs communs de l'agriculture durable et s'engagent dans l'organisation de la force ouvrière et l'utilisation collective des équipements et des infrastructures de production dans le processus total ou partiel 
(10) La reconnaissance de l'agriculture communautaire figure dans la loi fondamentale relative à l'alimentation, l'agriculture et les régions rurales (1999). de la production agricole, y compris de transformation, de stockage et de vente ». D'un point de vue organisationnel, chaque habitant désireux de former et de rejoindre une communauté agricole doit signer l'« accord de l'agriculture communautaire » après des réunions et des discussions intensives. Après la mise en pratique de cet accord, le groupe peut être reconnu comme organisation d'agriculture communautaire.

L’essence de la communauté agricole réside dans la préservation des terres agricoles et la restructuration de la riziculture par des méthodes innovantes, en mobilisant les ressources de production. En laissant la propriété des fermes agricoles à chaque membre, la communauté obtient les droits d'utilisation des parcelles qui sont nécessaires ou souhaitables pour la consolidation, afin d'atteindre une utilisation efficiente du terrain dans son ensemble. Cela permet, dans une certaine mesure, à la communauté de bénéficier au moins des économies d'échelle. L'utilisation coopérative des machines et de la main-d'œuvre augmente la productivité. La main-d'œuvre agricole est plus concentrée sur les exploitations agricoles ayant l'expérience du travail. En retour, elles obtiennent une compensation équitable. Les fermiers à temps partiel et ceux qui ont abandonné l'agriculture commerciale peuvent rester membres. Tout en recevant un loyer pour leurs parcelles, ces derniers peuvent occuper d'autres emplois dans le management, le travail administratif et de bureau ou peuvent être bénévoles durant les périodes de plantation et de récolte ou pour des travaux agricoles plus légers. Tous les membres doivent cependant travailler collectivement afin de maintenir leurs ressources communes telles que les routes, les cours d'eau, les réservoirs et l'entretien des paysages (Satoyama). La préservation des ressources naturelles locales et la prévention de la destruction de l'environnement sont ainsi devenues un enjeu important.

\section{INSTITUTIONNALISATION DE L'AGRICULTURE COMMUNAUTAIRE}

Le gouvernement a reconnu l'agriculture communautaire en $1999^{(10)}$. Depuis 2007, le ministère de l'Agriculture, des Forêts et de la Pêche (MAFP) soutient ces organisations, sous certaines conditions. La reconnaissance formelle était un avantage mitigé, car de nombreuses tentatives d'agriculture communautaire sont apparues uniquement pour bénéficier des nouvelles subventions. En outre, la politique du gouvernement a mis exclusivement l'accent sur la consolidation des exploitations agricoles afin d'accroître le travail agricole et de le rendre plus compétitif. Alors que le gouvernement encourageait les organisations d'agriculture communautaire à constituer une entité avec personnalité morale, il ne leur a pas octroyé de statut corporatif. Afin de devenir une entité avec personnalité 
morale, l'organisation devait adopter l'une des formes légales des entreprises existantes, telles que les sociétés coopératives de producteurs agricoles (CPA) et les sociétés de droit commun (entreprises par actions, sociétés à responsabilité limitée, partenariats à responsabilité limitée).

Le nombre d'organisations d'agriculture communautaire a augmenté. Selon la dernière étude du MAFP, publiée en février 2014, il existait 14717 organisations d'agriculture communautaire au niveau national, dont $22,1 \%$ avaient une personnalité juridique. Par le statut corporatif, la majorité (76,7\%) étaient des entreprises coopératives de producteurs agricoles (SCPA). Parmi les autres (23,3\%), $13 \%$ étaient des entreprises par actions, le reste ayant d'autres formes (sociétés en nom collectif, sociétés à participation limitée, entreprises à responsabilité limitée). Au total, l'agriculture communautaire a consolidé 49,2 millions d'hectares de terres agricoles.

\section{EVOLUTION DE L'AGRICULTURE DANS LES ACTIVITÉS COMMERCIALES ET STRUCTURES ORGANISATIONNELLES Diversification des activités agricoles et création des entreprises communautaires}

Les activités économiques des organisations d'agriculture communautaire se sont rapidement diversifiées pour inclure des activités telles que la vente et la transformation, créant ainsi des liens en amont et en aval. Auparavant, les agriculteurs comptaient exclusivement sur les marchés de gros locaux, ou Nokyo (AJ), pour la vente et la transformation de leurs produits, mais ils ont décidé de prendre en main ces activités. En agissant de la sorte, ils ont pu internaliser une partie importante de la valeur ajoutée. Les ventes directes sous la forme de stands de produits alimentaires locaux (Chokubai-Sho) et de marchés routiers (Michi-no-Eki) se sont rapidement répandues dans différentes régions rurales. Cette diversification a ensuite concerné le secteur des services (restaurants, écoles de cuisine, livraison de nourriture et tourisme vert), par l'établissement d'un lien direct avec les consommateurs ou avec des petites et moyennes entreprises locales.

Il faut souligner à ce stade le rôle significatif joué par les femmes, dont certaines se sont engagées dans la production de denrées autres que le riz (légumes, fruits, champignons, fleurs...), créant ainsi leur propre coopérative de producteurs. Beaucoup d'autres ont toutefois choisi la vente et la transformation liées à l'agriculture. La transformation alimentaire, la vente directe de produits agricoles et d'aliments transformés ainsi que les restaurants ruraux sont l'activité économique majoritaire entreprise par les femmes rurales (Seki, Matsunaga, 2012).

Plus récemment, l'entrepreneuriat féminin a augmenté dans le domaine des échanges ruraux-urbains (restaurant de ferme, 
(11) Après la guerre, avec un niveau plus important d'éducation et grâce à l'amélioration de leurs droits civiques, les femmes se sont engagées dans des «mouvements pour l'amélioration de la vie rurale", considérés comme l'une des plus importantes initiatives pour l'amélioration des conditions de vie rurales dans l'Asie de l'après- guerre. tourisme dans les fermes, hébergement, etc.). De telles activités constituent "l'entreprise communautaire", qui offre aux femmes rurales des opportunités précieuses pour générer des revenus et être autosuffisantes. Les femmes sont en effet devenues des acteurs majeurs dans ces activités ${ }^{(11)}$.

Alors que l'approche du développement endogène, fondée sur la communauté, montre l'importance du capital social, dans ces diverses formes «la préoccupation majeure est la compatibilité entre cette création de ressources "collective" et la participation et l'insertion sociale des individus exclus" (Shucksmith, 2003). L'apparition de l'entrepreneuriat féminin dans les régions rurales était cruciale pour faire face à cette problématique.

\section{Services communautaires et autres activités}

Durant les années 2000, le vieillissement rapide de la population a entraîné une augmentation des demandes concernant la santé, l'aide aux personnes âgées et d'autres prestations sociales, tandis que l'influence traditionnelle de la famille et des associations locales a considérablement faibli. Les autorités locales, souvent accablées par des dettes et les contraintes budgétaires, ont réduit les offres de services publics essentiels. Le déclin de la population et de la clientèle a aussi forcé les cliniques locales, les magasins, les restaurants, les bus et les taxis, ainsi que d'autres services fournis par le secteur privé, à cesser leur activité. La fusion municipale (Heinsei), entre 1999 et 2009, et la fermeture des branches (ou filiales) des AJ (Nokyo), en raison d'un mouvement de fusion de grande envergure, ont eu un impact sérieux sur le maintien de la vie rurale. Les plus grandes infrastructures publiques, telles que les écoles primaires, les hôpitaux, les centres communautaires, ont fermé ou fusionné. Alors que les magasins de détail des AJ, qui sont devenus les seuls de ce genre, ont mis fin à leur service, les habitants ont été confrontés au manque de commerces pour leurs achats quotidiens.

Les individus et groupes de citoyens, majoritairement des femmes, ont répondu à ces défis en créant leurs propres "commerces communautaires" de nourriture ou de services quotidiens. Dans la préfecture de Shimane, les organisations d'agriculture communautaire se sont engagées dans de nombreux services tels que la livraison de nourriture, le service funéraire, les stations d'essence, les taxis, la gestion des équipements publics et des ateliers protégés. La majorité de ces organisations œuvrent dans le recrutement et la formation des nouveaux entrants dans l'agriculture, la protection de l'environnement et la préservation de la culture traditionnelle. La préfecture a mis en œuvre un projet afin d'encourager «la contribution des organisations d'agriculture communautaire pour la communauté» (2011-2013). 


\section{Changement dans la structure organisationnelle et la gouvernance}

Alors que l'entreprise coopérative de producteurs agricoles était adéquate pour la production coopérative et la gestion dans l'agriculture, les structures éligibles étaient légalement limitées à l'agriculture et aux activités liées. Ainsi, lors de l'expansion de leurs activités, les organisations d'agriculture communautaire devaient adopter d'autres formes juridiques. La plupart ont créé une entreprise grâce aux investissements des résidents, en plus de la SCPA existante, ou ont adopté le statut d'entreprise. Cela leur a permis plus de flexibilité. Lorsque certains résidents, membres ou non membres de la SCPA, créaient une entreprise communautaire, une collaboration avec ce groupe avait lieu. Comme le décrit le schéma 1, les étapes les plus importantes de l'agriculture communautaire ont été accompagnées par un changement dans la structure organisationnelle interne et par l'expansion des relations externes.

\section{Schéma 1}

\section{Evolution des organisations d'agriculture communautaire}

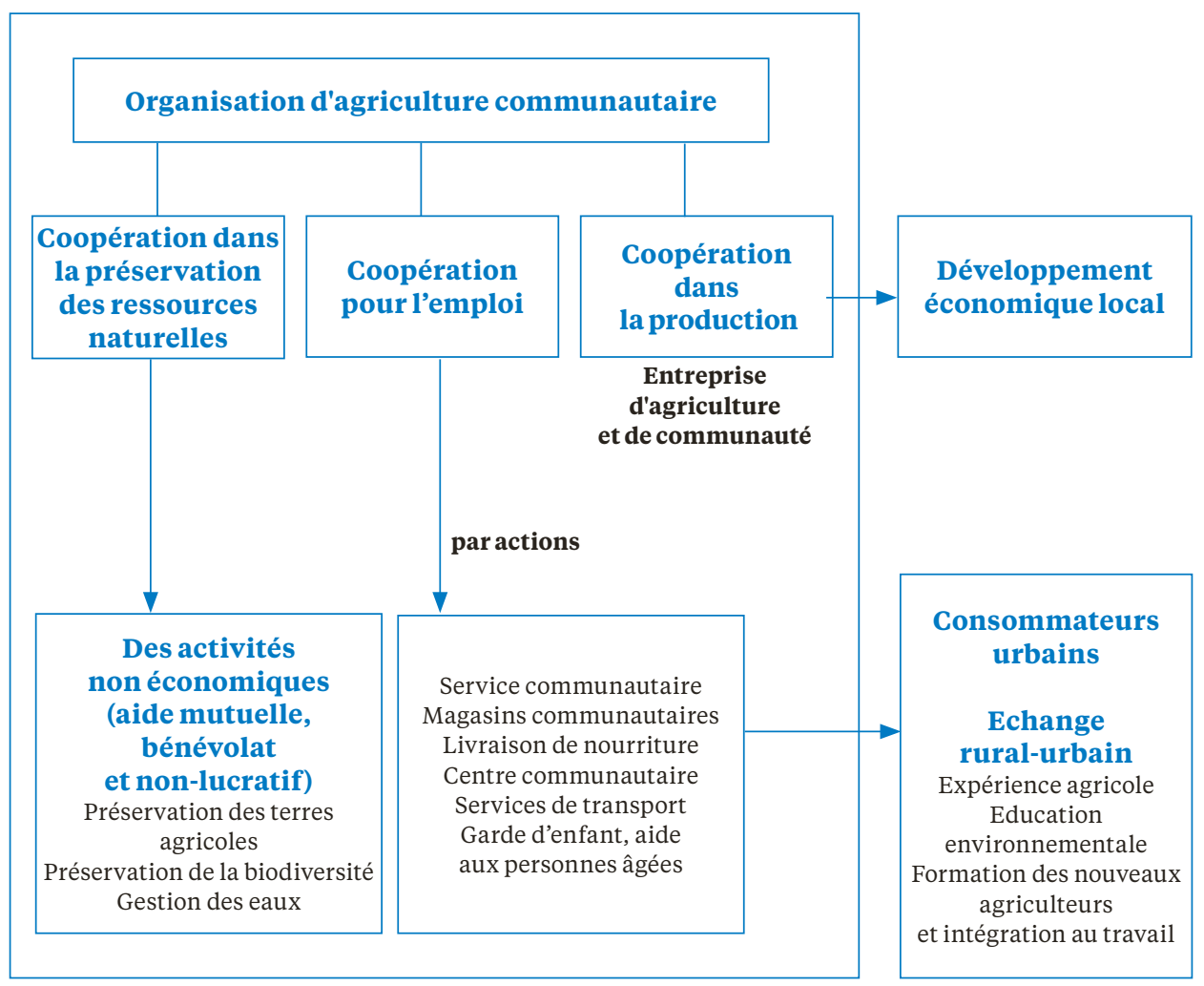


(12) Les districts patrimoniaux sont régis comme des collectivités locales spéciales par la loi sur l'autonomie locale.

\section{Initiatives locales des associations communautaires autonomes}

\section{FUSION " HEISEI " ET NAISSANCE DES ASSOCIATIONS COMMUNAUTAIRES AUTONOMES}

La fusion des municipalités Heisei, le regroupement national et la dissolution des municipalités (communes, villages, villes) qui a eu lieu entre 1999 et 2009 ont particulièrement touché les régions rurales. Le nombre de villes (Cho) et de villages (Son) représentant les régions rurales a considérablement baissé, de près de deux tiers (de 2558 à 935). De plus, $85 \%$ de ces anciennes municipalités se trouvaient dans des régions éloignées, montagneuses ou insulaires. Cette fusion visait par conséquent à la restructuration de ces régions désavantagées. Pourtant, un rapport d'évaluation publié en 2009 par l'Association nationale des villes et villages a identifié les problèmes de la fusion comme résultant de « la perte de dynamisme, de la perte de la tradition et de la culture, le déclin des services publics, et une voix plus faible des citoyens".

Afin de soutenir la fonction de maintien des ressources naturelles et du capital, des initiatives locales ont émergé, créant des associations communautaires d'autonomie locale (Chiiki jichi-soshiki). Comme le mot « autonomie » l'indique, l'association communautaire autonome représente en principe la communauté dans son ensemble selon un processus démocratique et elle assume des fonctions publiques en partenariat avec l'administration municipale. Parfois, un district et ses représentants servaient d'interface entre l'association communautaire d'autonomie locale et l'administration municipale. Le district, dans ce contexte, est une entité légale imitant un district patrimonial traditionnel ${ }^{(12)}$. Il peut acquérir de l'ancienne unité administrative (ville ou village) certains biens de valeur tels que les centres culturels et assurer des fonctions administratives, dans certaines limites. Son représentant doit être élu par les habitants.

Les associations communautaires autonomes étaient actives dans la plupart des préfectures au Japon, suite à la fusion Heisei. En cas de pérennité, l'association communautaire autonome se transforme en une organisation centrale de la communauté, agissant comme un organisme de coordination entre les coopératives, les entreprises et les différents organismes locaux, en assumant des fonctions économiques et non économiques. Les fonctions économiques comprennent l'investissement dans les entreprises appartenant aux résidents et le lancement de leurs propres entreprises et services communautaires, tels que la gestion des centres communautaires et des parcs, la création et l'exploitation de musées culturels et historiques, le tourisme vert et les services de transport locaux.

Parmi les exemples les plus anciens de réussite d'associations communautaires autonomes, il y a les conseils de promotion communautaires de Kawane dans la ville de Takamiya 
(préfecture d'Hiroshima) et ceux de Miyama (préfecture de Kyoto), tous deux apparus avant la fusion Heisei. L'une des leçons les plus importantes des exemples de Kawane et Miyama est le principe organisationnel et opérationnel du conseil de promotion communautaire et le partenariat solide avec le bureau municipal. Tout d'abord, ces conseils ont bénéficié de l'aide pratique et de l'expertise de l'administration municipale, indépendamment des associations locales. Cette discontinuité les a amenés à rompre avec la répartition traditionnelle des pouvoirs dans les villages, pour établir une gouvernance démocratique. Par ailleurs, l'administration municipale a encouragé le processus d'autodétermination et d'autogouvernement des habitants. Ce processus a donné le pouvoir aux conseils et leur a permis de ne pas devenir une sorte de sous-traitants des autorités locales, mais des associations autonomes pour la résolution de problèmes, traitant à égalité avec les autorités municipales pour proposer et mettre en œuvre des politiques publiques, et pgérer des projets économiques de façon autonome.

\section{LES CONSEILS DE PROMOTION COMMUNAUTAIRE DE MIYAMA}

Miyama est une ville montagneuse et isolée dans la préfecture de Kyoto. Elle représente un bon exemple de l'évolution de l'agriculture communautaire, et du rôle de l'association communautaire d'autonomie locale et des partenariats public-privé. Miyama a connu un dépeuplement et un vieillissement rapides, passant de 10000 habitants en 1965 à 5000 au milieu des années 2000. La part des personnes âgées ( 65 ans et plus) dans la population totale était de $32,6 \%$ en 2001, presque deux fois la moyenne nationale (17\%). La ville est cependant riche en ressources culturelles, avec notamment 250 maisons japonaises traditionnelles aux toits de chaume préservées depuis des siècles.

L'évolution de la communauté endogène de Miyama peut être divisée en quatre étapes. La première (1978-1988) était caractérisée par la promotion de l'agriculture communautaire. Des initiatives de transformations agricoles et de commercialisation des produits locaux ont été engagées avec les habitants. La deuxième phase (1989-1992) fut celle des échanges actifs ruraux-urbains et de la régénération communautaire. Le Fonds de préservation des maisons traditionnelles de la ville de Miyama est né, avec la participation d'intellectuels de la région urbaine du district de Kansai (majoritairement Osaka et Kobe). De nombreuses actions de préservation des ressources culturelles locales et de redécouverte du patrimoine ont eu lieu. Durant la troisième phase (1993-1998), Miyama a développé l'écotourisme et d'autres industries de services qui y sont liées. Elle a présenté un plan visant à créer des espaces d'habitation confortables en zone rurale pour l'installation de repreneurs d'exploitations agricoles. 
Par ailleurs, des organisations non lucratives et des groupes de bénévoles, parmi lesquels de nombreuses femmes, ont lancé de multiples activités, particulièrement après le tremblement de terre de Hanshin-Awaji (1995).

La quatrième phase, depuis 1999, est marquée par le rôle actif des conseils de promotion communautaire. Suite au regroupement de grande envergure des coopératives agricoles AJ, la branche AJ de Miyama a été supprimée en 1999, puis celles qui exploitaient uniquement les commerces de détail dans chaque district ont été fermées. Avec des fonds d'investissement de la communauté, un groupe de résidents a repris et relancé ces magasins sous le statut d'entreprises à responsabilité limitée (quatre entreprises entre 1999 et 2002). Ces entreprises se sont lancées ensuite dans la conservation des terres agricoles et l'action sociale. En 2000, le conseil de ville de Miyama a proposé la mise en place de conseils de promotion communautaire dans ses cinq districts (auparavant des villages administratifs) en regroupant les trois quartiers existants et les organisations communautaires. Les conseils partageaient un objectif commun: "devenir l'Inaka (campagne) numéro un du Japon ", en améliorant la qualité de vie des résidents.

Les conseils de promotion communautaire sont nés des partenariats public-privé entre la municipalité et les organisations de résidents, ce qui constitue une approche nouvelle de l'autonomie et de la coopération par la réhabilitation des vieux villages. Le conseil municipal de Miyama a proposé un soutien financier et humain, en détachant du personnel administratif à chaque conseil. En 2006, Miyama a été regroupé avec trois autres villes au sein de la ville de Nantan, et les cinq conseils de promotion communautaire se sont regroupés au sein d'une alliance. Le comité de développement communautaire de Miyama, qui est centré sur des fonctions de planification et de défense pour toute la ville, est composé de citoyens actifs de chaque conseil de district. Les caractéristiques du partenariat et la structure de gouvernance du conseil de promotion communautaire de chaque district sont cependant restées globalement les mêmes.

La ville a réussi à tirer parti de ses ressources naturelles et culturelles abondantes. En 2010, par exemple, elle a attiré environ 700000 touristes de la région voisine du Kansai et de l'étranger (majoritairement de Taïwan et de Chine populaire). Malgré les efforts des conseils de promotion communautaire, des autres organisations et des citoyens, le bilan migratoire a rapidement décliné et le vieillissement s'est accentué. La création de capacités et le développement des ressources humaines pour les héritiers de la première génération de militants ainsi que la mobilité de la jeune génération sont des défis urgents. 


\section{Les trois catégories d'entreprises sociales rurales : similarités et différences}

Au regard de l'approche conceptuelle de l'entreprise sociale de l'EMES, il convient de s'interroger pour savoir si les entreprises rurales communautaires peuvent y entrer, car elle s'ancre profondément dans la tradition européenne d'économie sociale. Contrairement à l'approche «tiers secteur » non lucratif aux Etats-Unis, elle identifie l'entreprise sociale en tant que nouvelle forme de dynamique entrepreneuriale au cœur même de l'économie sociale. L'entreprise sociale vient essentiellement en réponse à des besoins non pris en charge ou d'une manière insuffisante par le secteur public et les entreprises à but lucratif (Defourny, Nyssens, 2013).

Nos résultats montrent qu'il existe de nombreuses entreprises sociales dans les régions rurales, dans le sens où elles sont apparues en tant qu'organisations de l'économie sociale existante, mais redéfinies par de nouvelles dynamiques entrepreneuriales. Le tableau 1 (en page suivante) fournit un résumé des trois types d'organisations ou des catégories d'entreprises rurales décrites dans la deuxième partie. Ces trois catégories sont apparues dans différents endroits et périodes, dans un contexte historique et par des initiatives uniques.

L'approche de Defourny et Nyssens (2012) inclut la définition de l'entreprise sociale, avec trois dimensions: la dimension économique et entrepreneuriale, la dimension sociale (la primauté de l'objectif social) et la gouvernance participative. Chaque dimension comprend trois ensembles d'indicateurs. Dans le cadre d'une coopérative, qui présente la plupart des caractéristiques clés de l'entreprise sociale, les principaux éléments de différenciation avec les organisations traditionnelles de l'économie sociale résident dans la nature de l'innovation sociale, y compris les dynamiques de leurs structures de gouvernance.

Concernant la première catégorie de coopératives de producteurs et de fermes dans l'agriculture biologique ou naturelle, leur mission sociale, initialement limitée à la santé et la sécurité alimentaire, s'est étendue à la revitalisation de l'agriculture locale et de la communauté par une agriculture durable. Bien qu'elles énoncent une variété de fonctions et d'objectifs, elles restent essentiellement liées à l'agriculture. Ainsi, d'un point de vue conceptuel, nous pouvons considérer ces entreprises comme « unifonctionnelles ». Concernant l'innovation sociale, le Teikei original a été une méthode pionnière pour réunir des producteurs et des consommateurs, et valoriser la coproduction basée sur la confiance et le soutien mutuel. Plus tard, cette même méthode fut étendue aux organisations, instaurant le même type de relation entre, par exemple, des coopératives de producteurs et des coopératives de consommateurs ou, dans le commerce équitable, 


\section{Tableau 1}

\section{Panorama des entreprises rurales communautaires}

\begin{tabular}{|c|c|c|c|}
\hline $\begin{array}{l}\text { Type } \\
\text { d'organisation }\end{array}$ & $\begin{array}{l}\text { Ferme ou coopérative } \\
\text { de producteurs biologiques } \\
\text { et naturels }\end{array}$ & $\begin{array}{l}\text { Société d'agriculture } \\
\text { fondée sur la communauté }\end{array}$ & $\begin{array}{l}\text { Association } \\
\text { communautaire } \\
\text { autonome }\end{array}$ \\
\hline $\begin{array}{l}\text { Initiative } \\
\text { et année } \\
\text { de création }\end{array}$ & $\begin{array}{l}\text { Un individu } \\
\text { ou un groupe d'individus } \\
1970 \text { début des années } 1980\end{array}$ & $\begin{array}{l}\text { Un groupe d'habitants de la } \\
\text { communauté (ex. un village) } \\
1990 \text { et } 2000\end{array}$ & $\begin{array}{l}\text { Habitant de la communauté } \\
\text { et municipalité } \\
2000\end{array}$ \\
\hline $\begin{array}{l}\text { Contexte, } \\
\text { raison d'être }\end{array}$ & $\begin{array}{l}\text { Nouvelle coopérative } \\
\text { et mouvements citoyens } \\
\text { Dégradation de l'environnement, } \\
\text { produits alimentaires } \\
\text { dangereux, production } \\
\text { et consommation non durables }\end{array}$ & $\begin{array}{l}\text { Agriculture communautaire } \\
\text { Problèmes dans l'agriculture, } \\
\text { déclin du rôle } \\
\text { des coopératives agricoles } \\
\text { classiques (Nokyo ou AJ) }\end{array}$ & $\begin{array}{l}\text { Réponse à la désagrégation } \\
\text { de la population } \\
\text { Déclin des fonctions } \\
\text { communautaires, Heisei } \\
\text { (fusion des municipalités) }\end{array}$ \\
\hline Site principal & $\begin{array}{l}\text { Des régions productrice de riz } \\
\text { dans le Nord-Est, le Nord, l'Est }\end{array}$ & $\begin{array}{l}\text { Régions vallonnées } \\
\text { et montagneuses } \\
\text { désavantagées dans l'Ouest }\end{array}$ & $\begin{array}{l}\text { Régions vallonnées } \\
\text { et montagneuses } \\
\text { désavantagées }\end{array}$ \\
\hline $\begin{array}{l}\text { Modèle } \\
\text { d'entreprise } \\
\text { sociale }\end{array}$ & Coopérative communautaire & $\begin{array}{l}\text { Coopérative communautaire } \\
\text { (coopérative sociale ou } \\
\text { coopérative d'intérêt public) }\end{array}$ & $\begin{array}{l}\text { Coopérative communautaire } \\
\text { (société de développement } \\
\text { communautaire) }\end{array}$ \\
\hline Mission sociale & $\begin{array}{l}\text { Revitalisation de l'agriculture } \\
\text { locale par l'agriculture durable }\end{array}$ & $\begin{array}{l}\text { Protection des terres } \\
\text { agricoles, revitalisation de } \\
\text { l'agriculture locale et garantie } \\
\text { des moyens de subsistance }\end{array}$ & $\begin{array}{l}\text { Redynamisation } \\
\text { de la communauté } \\
\text { pour garantir les moyens } \\
\text { de subsistance }\end{array}$ \\
\hline $\begin{array}{l}\text { Objectifs, } \\
\text { fonctions }\end{array}$ & $\begin{array}{l}\text { Agriculture durable } \\
\text { Sécurité alimentaire et santé } \\
\text { Protection des ressources } \\
\text { naturelles. } \\
\text { Production coopérative }\end{array}$ & $\begin{array}{l}\text { Coopérative agricole } \\
\text { Entreprise communautaire } \\
\text { Services communautaires } \\
\text { Protection des ressources } \\
\text { naturelles }\end{array}$ & $\begin{array}{l}\text { Développement } \\
\text { de la communauté } \\
\text { Renforcement des ressources } \\
\text { et services communautaires }\end{array}$ \\
\hline $\begin{array}{l}\text { Types } \\
\text { d'innovation } \\
\text { sociale }\end{array}$ & $\begin{array}{l}\text { Teikei et agriculture } \\
\text { soutenue parlacommunauté } \\
\text { Coproduction } \\
\text { producteur-consommateur } \\
\text { Commerce équitable } \\
\text { rural-urbain } \\
\text { Réalisation d'un système } \\
\text { d'alimentation locale } \\
\text { par l'agriculture durable }\end{array}$ & $\begin{array}{l}\text { Agriculture communautaire } \\
\text { Mobilisation et organisation } \\
\text { des ressources locales } \\
\text { Entrepreneuriat féminin } \\
\text { Création d'opportunité } \\
\text { de revenus pour les femmes } \\
\text { Action collective } \\
\text { Préservation des ressources } \\
\text { naturelles et de l'environnement }\end{array}$ & $\begin{array}{l}\text { Partenariat public-privé } \\
\text { Implication des différentes } \\
\text { parties prenantes } \\
\text { Engagement citoyen }\end{array}$ \\
\hline $\begin{array}{l}\text { Structure } \\
\text { de gouvernance }\end{array}$ & $\begin{array}{l}\text { Groupe d'entrepreneurs } \\
\text { sociaux, coopérative }\end{array}$ & $\begin{array}{l}\text { Coopérative ou groupe } \\
\text { de coopératives } \\
\text { dans la communauté }\end{array}$ & $\begin{array}{l}\text { Municipalité et conseil } \\
\text { autonome local, autres } \\
\text { organisations privées } \\
\text { ou associations de bénévoles }\end{array}$ \\
\hline $\begin{array}{l}\text { Réseaux } \\
\text { (collaboration } \\
\text { avec des } \\
\text { organisations } \\
\text { externes) }\end{array}$ & $\begin{array}{l}\text { Collaboration avec des groupes } \\
\text { de consommateurs et des } \\
\text { coopératives d'alimentation } \\
\text { naturelle, des coopératives } \\
\text { de travailleurs, organismes de } \\
\text { défense (local, national, étranger) } \\
\text { Collaboration avec } \\
\text { des entreprises locales, } \\
\text { les autorités locales (village, ville) }\end{array}$ & $\begin{array}{l}\text { Collaboration avec } \\
\text { les entreprises agricoles, les } \\
\text { organisations d'agriculture } \\
\text { communautaire, } \\
\text { des organisations } \\
\text { de quartier et de bénévoles, } \\
\text { les coopératives Nokyo (AJ), } \\
\text { les autorités locales } \\
\text { (village, ville) }\end{array}$ & $\begin{array}{l}\text { Organisme de coordination } \\
\text { pour les coopératives, } \\
\text { les entreprises, } \\
\text { les organisations civiques } \\
\text { et de citoyens } \\
\text { dans la communauté } \\
\text { Collaboration avec } \\
\text { les organisations non lucratives } \\
\text { et les universités }\end{array}$ \\
\hline Forme juridique & $\begin{array}{l}\text { SCPA, LLC spécial*, } \\
\text { sociétés par actions, fermes } \\
\text { non constituées en sociétés }\end{array}$ & $\begin{array}{l}\text { SCPA, LLC spécial*, sociétés } \\
\text { par actions }\end{array}$ & $\begin{array}{l}\text { Organisation des relations au } \\
\text { niveau local, association avec } \\
\text { ou sans personnalité morale }\end{array}$ \\
\hline
\end{tabular}

*LLC Spécial est un statut transitoire pour les anciennes « entreprises limitées », aboli par la loi des sociétés révisée en 2006. Elles peuvent rester des entreprises par actions non publiques avec des restrictions dans le transfert des actions. 


\footnotetext{
(13) L'utilisation de ce terme dans ce contexte peut être différent des coopératives sociales dans d'autres pays, comme en Italie ou en Corée du Sud, qui sont centrées sur l'intégration par l'emploi et l'activité. Puisque ces organisations coopératives présentent des réponses nouvelles et innovantes aux besoins urgents de la communauté, nous pouvons cependant les appeler « coopératives sociales » ou coopératives d'intérêt public.
}

entre ruraux et urbains. La contribution des Teikei à ce stade porte essentiellement sur l'élargissement de la notion d'" agriculture soutenue par la communauté » ou des systèmes d'alimentation alternative fondés sur la communauté.

Dans la deuxième catégorie, les coopératives agricoles issues de l'agriculture communautaire ont progressivement évolué vers des entreprises et services communautaires: elles sont devenues multifonctionnelles. Leur objectif initial de préservation des terres agricoles a par conséquent évolué vers la prise en compte du développement de l'économie locale et vers la garantie des moyens de subsistance de la communauté. L'innovation sociale de ce type peut être caractérisée par les quatre points suivants : - un moyen efficient et équitable de mobiliser et d'organiser les ressources de production, telles que les terres agricoles, le capital, la main-d'œuvre (agricole ou non agricole, rémunérée ou non rémunérée);

- la création d'opportunités de revenu et d'emploi grâce à des entreprises communautaires et la mise en place de services essentiels du quotidien (services communautaires) dans les régions désavantagées;

- l'entrepreneuriat féminin et l'empowerment des femmes grâce à des entreprises communautaires et à des services communautaires;

- l'action collective dans la protection des ressources naturelles, y compris les réserves communes, l'environnement et la biodiversité. Kusumoto (2010) décrit les "cas les plus avancés d'organisations d'agriculture communautaire » comme ayant trois piliers de coopération (Kyodo): la coopération dans la production, la coopération dans la protection des ressources naturelles et de l'environnement, et la coopération pour les moyens de subsistance. Kusumoto considère ainsi ces organisations comme une nouvelle forme de "coopérative sociale ${ }^{(13)}$ ».

Le troisième et dernier modèle de l'" association communautaire autonome » est le plus inclusif concernant la mission de l'organisation, les fonctions et la gouvernance. La redynamisation de la communauté, par la fourniture de services économiques et d'intérêt général, est leur raison d'être. La majorité de leurs projets économiques ont un caractère public et peuvent donc concerner le développement de l'écotourisme, la gestion des centres communautaires et la formation continue, les musées et commerces culturels et historiques, la vente des spécialités de la communauté et les transports en commun. Ces associations sont inévitablement liées à d'autres fonctions non économiques, comme la préservation du patrimoine historique, naturel et culturel de la communauté et le développement des ressources humaines. L'innovation sociale de ce genre d'organisations se caractérise par le renforcement des capacités de la communauté grâce à des partenariats public-privé, 
l'implication de nombreuses parties prenantes et l'engagement des citoyens. Ainsi, nous pouvons décrire ce nouveau type particulier d'entreprise sociale comme «société de développement communautaire».

Ces modèles ne s'excluent pas mutuellement. Si nous pouvons les distinguer d'un point de vue conceptuel, cela est souvent impossible d'un point de vue empirique. Par exemple, le conseil de préservation des terres agricoles, de l'eau et de l'environnement de Shimosato, que nous avons classé dans le premier modèle de l'agriculture soutenue par la communauté, peut aussi être considéré comme de l'agriculture communautaire. Comme dans le cas des conseils de promotion communautaire de Miyama, le modèle de "société de développement communautaire " a émergé comme une évolution de l'agriculture communautaire ou comme une forme d'agriculture communautaire combinée avec de nouvelles organisations à but social.

Concernant les formes juridiques, il n'existe pas de statuts d'entreprise adaptés à ces trois types d'entreprises rurales. Actuellement, elles empruntent les formes juridiques existantes. Les deux premiers modèles ont fréquemment adopté les formes de sociétés coopératives de producteurs agricoles (SCPA), sociétés anonymes spéciales et sociétés par actions. La dernière option est de plus en plus utilisée, car les démarches administratives pour le changement des SCPA en sociétés par actions ont été allégées en 2005. Dans le cas d'associations communautaires autonomes, qui sont des organisations multifonctionnelles avec plusieurs parties prenantes, certaines relèvent de la loi sur l'autonomie locale et d'autres sont des organisations non lucratives. Nombre d'entre elles sont restées sans personnalité morale, car aucun statut légal n’est adéquat pour ce type d'organisation.

Ainsi, s’il existe des similarités et des différences entre ces modèles, les distinctions sont brouillées sur certains éléments importants. Une caractéristique importante de ces entreprises est en effet leur effort d'innovation et leur structure organisationnelle. Leur caractère évolutif est à dissocier de leur statut de société, qui est relativement restrictif, segmenté et strictement réglementé par la loi. Malgré les différences d'origine et de forme juridique, les entreprises répondent toutes, d'une manière ou d'une autre, aux besoins urgents des communautés rurales confrontés à la crise actuelle dans l'agriculture. Les modèles les plus aboutis de ces entreprises, créées à l'initiative d'un groupe de citoyens, visent à la redynamisation de la communauté et au développement durable. "Redécouvrant » et mobilisant les ressources de la communauté et ses actifs de catégories différentes (physique, financier, humain, naturel [culturel] et capital social), ils fournissent différents types de «biens communautaires » parapublics avec un modèle évolutif d’autogouvernance. 
De plus, la communauté devient plus ouverte et inclusive. En ce sens, les trois modèles peuvent être décrits comme " coopératives communautaires ".

\section{Conclusion}

Si la littérature sur l'économie sociale et solidaire est riche en travaux de recherche sur les coopératives, notamment dans l'agriculture (Defourny, Nyssens, 2012), il existe cependant peu d'études concernant les modèles d'entreprises coopératives au sein des communautés rurales dans le cadre de la globalisation et des changements sociaux importants. Notre étude et nos recherches futures ont pour but de combler cet écart.

Mori (2014) discute les perspectives des « coopératives communautaires », à partir de l'histoire des coopératives d'énergies renouvelable (REScoops) et d'autres orientées vers la communauté en Italie, au Royaume-Uni et dans d'autres pays européens. Il identifie trois éléments fondamentaux de ce concept: un territoire clairement défini, des biens communautaires, qui sont les services d'intérêt général pour tous les résidents, et la citoyenneté, ce qui sous-entend que les projets économiques appartiennent à la communauté et que les citoyens y prennent une part active.

Les résultats de notre étude tendent à appuyer l'hypothèse selon laquelle le dernier modèle d'entreprise rurale au Japon forme un nouveau modèle d'entreprise sociale qui peut être appelé "coopérative communautaire", comme défini par Mori. Actuellement, au Japon, de façon générale, il n’existe aucune forme juridique pour les entreprises sociales, ni pour les nouvelles formes d'entreprises telles que les coopératives d'énergie et les coopératives multifonctionnelles et multiparties prenantes. Les tentatives de légalisation des entreprises sociales communautaires, comme celles discutées dans cette étude, ont abouti en 2009 au projet de loi fondamentale sur les activités communautaires et l'intégration des conseils communautaires autonomes. Ces projets n'ont pas avancé ultérieurement à cause des changements de majorité gouvernementale, mais la discussion se poursuit sur le terrain académique, en relation avec les acteurs. 


\section{BIBLIOGRAPHIE}

Defourny J., Nyssens M., 2010, « Conceptions of social enterprise and social entrepreneurship in Europe and the United States: convergences and divergence", Journal of Social Entrepreneurship, vol. 1, $\mathrm{n}^{\circ} 1, \mathrm{p} .32-53$.

Defourny J., Nyssens M., 2012, « The EMES Approach of Social Enterprise in a Comparative Perspective ", Working Paper, $\mathrm{n}^{\circ} 12 / 03$, EMES European Research Network.

Defourny J., Nyssens M., 2013, "Social innovation, social economy and social enterprise: What can the European debate tell us? », in Moulaert et al., eds.

Iwami H., 2007, Social History of Workers Cooperatives in Japan, Tokyo, Ryokufu Shuppan.

Kusumoto M., 2010, Community Farming Evolving, Tokyo, Noubunkyo.
Mori P.A., 2014, « Community and Cooperation: The Evolution of Cooperatives towards New Models of Citizen's Democratic Participation in Public Services Provision ", Euricse Working Paper, $\mathrm{n}^{\circ}$ 63/14.

O’Hara J.K., 2011, « Market Forces: Creating Jobs through Public Investment in Local and Regional Food Systems ", Boston, $M A$ : Union of Concerned Scientists.

Seki M., Matsunaga K., 2012, Community farming: Opening An Avenue for the Future, Shin Hyoron.

Shimane Prefecture, 2002, "Towards the promotion of new community farming ».

Shucksmith M., 2003, "Endogenous development, social capital and social inclusion: perspectives from Leader in the USA », Sociologia Ruralis, n 40, p. 208-218. 